\title{
Genome survey and high-resolution backcross genetic linkage map construction of the ridgetail white prawn Exopalaemon carinicauda applications to QTL mapping of growth traits
}

\author{
Jitao $L i^{1,2}$, Jianjian $L v^{1,2}$, Ping Liu ${ }^{1,2}$, Ping Chen ${ }^{1,2}$, Jiajia Wang ${ }^{1,2}$ and Jian $L i^{1,2^{*}}$
}

\begin{abstract}
Background: High-resolution genetic linkage map is critical for QTL mapping, genome sequence assembly and marker-assisted selection in aquaculture species. The ridgetail white prawn Exopalaemon carinicauda is one of the most economic shrimp species naturally distributed in the coasts of eastern China and western Korea. However, quite limited genomics and genetics information have been exploited for genetic improvement of economic traits in this species.

Results: In the present study, we conducted genome survey and constructed high-resolution genetic linkage maps of the ridgetail white prawn with reciprocal-cross mapping family genotyped using next-generation sequencing approaches. The estimated genome size was $9.33 \mathrm{~Gb}$ with a heterozygosity of $0.26 \%$ and a repeat sequence ratio of $76.62 \% .65,772$ protein-coding genes were identified by genome annotation. A total of 10,384 SNPs were used to high-throughput genotyping and assigned to 45 linkage groups (LGs) from reciprocal backcross families of $E$. carinicauda, and the average marker distances were $0.73 \mathrm{cM}$ and $0.55 \mathrm{cM}$, respectively. Based on the high-resolution linkage map, twenty-three QTLs related to five growth traits were detected. All QTLs could explain 8.8-15.7\% of the total growth-traits variation.

Conclusions: The genome size of E. carinicauda was estimated more accurately by genome survey analysis, which revealed basic genomic architecture. The first high-resolution backcross genetic linkage map and QTLs related to growth traits will provide important information for QTL fine mapping, genome assembly and genetic improvement of E. carinicauda and other palaemon shrimps.
\end{abstract}

Keywords: Exopalaemon carinicauda, Genome, SNP, High-resolution linkage map, QTL mapping

\section{Background}

The ridgetail white prawn Exopalaemon carinicauda is an important economic shrimp species naturally distributed in the coasts of eastern China and western Korea [1]. Due to multiple advantages of fast growth, good

\footnotetext{
* Correspondence: lijian@ysfri.ac.cn

${ }^{1}$ Ministry of Agriculture and Rural Affairs, Yellow Sea Fisheries Research Institute, CAFS, Key Lab for Sustainable Development of Marine Fisheries, Qingdao 266071, China

${ }^{2}$ Function Laboratory for Marine Fisheries Science and Food Production Processes, National Lab for Ocean Science and Technology, Qingdao 266071, China
}

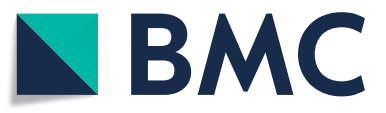

(c) The Author(s). 2019 Open Access This article is distributed under the terms of the Creative Commons Attribution 4.0 International License (http://creativecommons.org/licenses/by/4.0/), which permits unrestricted use, distribution, and reproduction in any medium, provided you give appropriate credit to the original author(s) and the source, provide a link to the Creative Commons license, and indicate if changes were made. The Creative Commons Public Domain Dedication waiver (http://creativecommons.org/publicdomain/zero/1.0/) applies to the data made available in this article, unless otherwise stated. environment, the aquaculture scale of the ridgetail white prawn expanded rapidly and contributed to one third of the total output of the polyculture ponds in eastern China [2]. The annual aquaculture area and yield of $E$. carinicauda in China are about 20, 000 ha and 45, 000 tons, respectively. But the aquaculture of E. carinicauda mainly relyed on natural seeds and wild spawning broodstock, which resulted in unclear genetic background and germplasm degradation, seriously affecting industrial development. Conventional breeding methods 
based on selection of individuals on phenotypic values. The approaches of using molecular markers for selection, or by both markers and other phenotypic data, named marker-assisted selection (MAS) [3] was first applied when the restriction fragment length polymorphisms (RFLP) were detected in economic species [4]. MAS is especially helpful for traits that are difficult to measure, exhibit low heritability and/or are expressed late in development process. Implementation of MAS requires DNA markers that are tightly linked to quantitative trait loci (QTL) for economic traits based on genetic linkage maps [5]. Therefore, MAS approaches is urgently required for sustainable development in culture of $E$. carinicauda.

High-resolution genetic linkage map is necessary for genome assembly, as well as for mapping QTL of economic traits [6]. In the past decade, genetic linkage map had been constructed using various molecular markers in multiple aquaculture species [5, 7-10], including Tilapia, Rainbow trout, Atlantic salmon, Asian seabass, Half tongue sole, Channel catfish, Common carp, Japanese flounder and many others. Linkage map provide essential tools for QTL localization and promote MAS process in multiple aquaculture species. For example, growth traits have been mapped in Rainbow trout [11], Asian seabass [12], Salmons [13]; Vibrio anguillarum and lymphocystis disease-resistant traits in Japanese flounder have been successfully mapped and applied to marker-assistant breeding [9, 14]; sex-determination traits have been localized by QTL mapping approaches in Half tongue sole [7], Tilapia [15] and Atlantic halibut [16]. Recently, High-resolution genetic linkage maps have been constructed in crustaceans, such as Kuruma prawn [17], Chinese shrimp [18], Black tiger shrimp [19], Pacific white shrimp [20], Swimming crab [21] and Chinese mitten crab [22]. In contrast, a high-resolution linkage map is urgently needed for genomic and genetic researches in the ridgetail white prawn.

Next-Generation Sequencing (NGS) technology and associated genotyping advancements have become widely used to implement de novo genome sequencing and highresolution linkage map construction in non-model species. In recent years, Specific-Length Amplified Fragment sequencing (SLAF-seq) has been successfully applied in large-scale de novo SNP discovery and genotyping in various species [23]. As a reliable, high throughput and double-barcode genotyping platform, SLAF-seq is fast, accurate and cost-effective for high-resolution linkage maps construction, such as sesame [24], soybean $[25,26]$ and cucumber [27]. For aquaculture species, a high-resolution linkage map, including 5,885 markers, was constructed in common carp using SLAF-seq with marker intervals of $0.68 \mathrm{cM}$ on average [23]. High-resolution genetic map of the Pacific white shrimp was also constructed using this method with an average marker distance of $0.7 \mathrm{cM}$ [20].
The main purposes of constructing high-resolution linkage map is to conduct the mapping of QTLs related to economic traits. Based on the high-resolution linkage map, several QTLs related to body length and body weight of shrimps were detected [20,22].

Constructing a genetic linkage map requires reference populations/families where molecular markers segregate [28]. Because common crustaceans reproduced one generation per year, the most of reference populations of shrimps and crabs were $F_{1}$ and $F_{2}$ families [17-22]. Backcross populations, derived by crossing the $F_{1}$ hybrid to one of their parents, are usually used to construct genetic linkage map and QTL mapping due to direct reflection of separation of $F_{1}$ gametes and higher mapping efficiency than $F_{2}$ population $[11,29]$. In the present study, we constructed reciprocal-cross backcross population of E. carinicauda for the first time based on the characteristics of multiple reproduction annually. We then conducted genome survey analysis and construction of a high-resolution linkage map to investigate the genomic and genetic architecture of E. carinicauda. Based on the high-resolution linkage map, QTL mapping was also conducted to detect markers related to growth traits.

\section{Results}

\section{Genome survey of E. carinicauda}

We constructed six $270 \mathrm{bp}$ DNA libraries with short paired-end and sequenced them by Illumina Hiseq 4000 platform. 294.46 Gb high-quality reads with $40.01 \%$ GC content were obtained in total after sequencing, which covered approximately 31.57 -fold the genome size of $E$. carinicauda. Sequencing raw data have been submitted to the Nucleotide database of NCBI with the accession number QUOF000000000.1. K-mer curve was obtained based on the frequencies of 23-mers (nucleotide strings with a length of $23 \mathrm{bp}$ ) within sequencing data (Fig. 1). K-mer analysis revealed that there was a peak at the Kmer depth of 24. The genome size of $E$. carinicauda was estimated as $9.33 \mathrm{~Gb}$ with remarkably low heterozygosity (0.26\%), which were possessed by approximately $76.62 \%$ repeat sequences (Table 1). The total length of assembled scaffolds was $9.18 \mathrm{~Gb}$, which covered approximately $98.40 \%$ of E. carinicauda genome (Table 2). Finally, we predicted 65,772 genes (Additional file 2: Table S2) based on sequencing contigs/scaffold.

\section{Features of the phenotypic traits}

The mean values of body length $(B L)$, body weight $(B W)$, carpace length $(C L)$, carpace width $(C W)$ and carpace height $(\mathrm{CH})$ in backcross family were $39.25 \pm 5.78$ $\mathrm{mm}, 0.91 \pm 0.41 \mathrm{~g}, 20.42 \pm 3.57 \mathrm{~mm}, 5.54 \pm 0.82 \mathrm{~mm}$ and $6.27 \pm 0.94 \mathrm{~mm}$, respectively (Additional file 1 : Table S1). And the average values of body length $(B L)$, body weight 


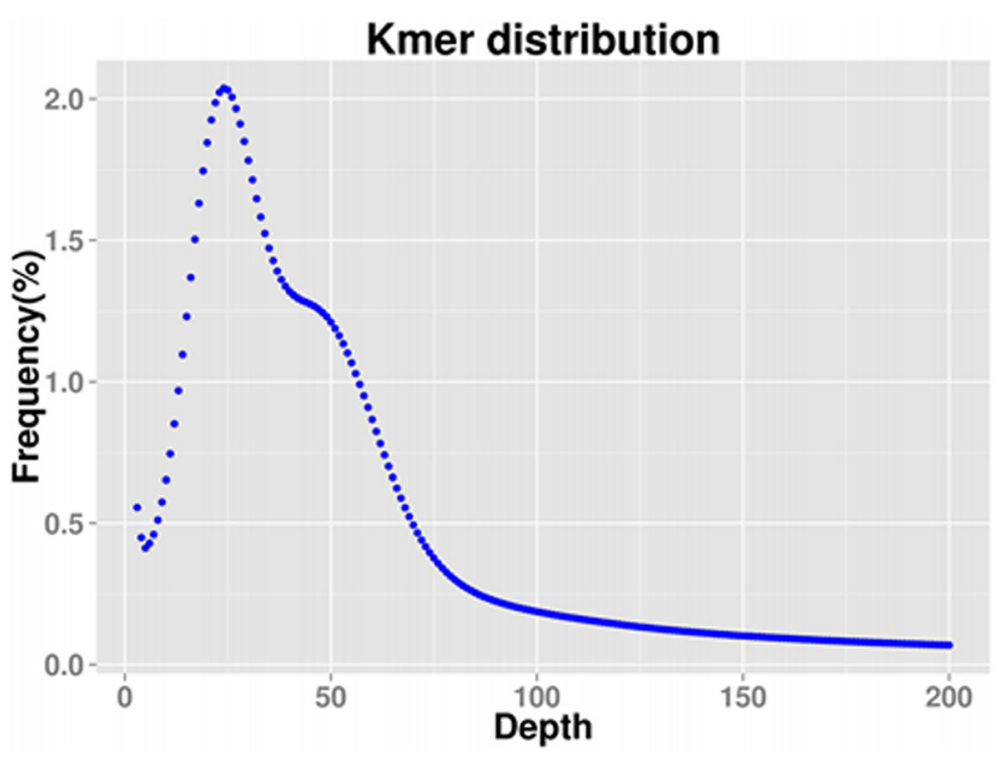

Fig. 1 Kmer distribution of E. carinicauda genome

$(B W)$, carpace length $(C L)$, carpace width $(C W)$ and carpace height $(\mathrm{CH})$ in reverse backcross family were $34.79 \pm 3.97 \mathrm{~mm}, 0.62 \pm 0.22 \mathrm{~g}, 18.20 \pm 2.56 \mathrm{~mm}, 4.88 \pm$ $0.62 \mathrm{~mm}$ and $5.56 \pm 0.60 \mathrm{~mm}$, respectively. The growthrelated traits showed strong correlations with each other ( $\mathrm{r}=0.774-0.983, P<0.001$ for all) (Tables 3 and 4$)$. The highest correlation value $(\mathrm{r}=0.983)$ was existed between $B L$ and $B W$. The $B W$ strongly correlated with $B L(\mathrm{r}=$ 0.983), $C H(\mathrm{r}=0.970)$ and $C W(\mathrm{r}=0.962)$.

\section{SLAF-seq library construction and sequencing}

A total of 204 SLAF-seq libraries from 4 parents and 200 backcross offsprings were constructed and sequenced on 4 lanes of Illumina HiSeq 2500 platform to generate 4.98 billion raw reads. After data trimming, $100.60 \mathrm{~Gb}$ of sequencing data, were individually divided into SLAF tags according to their MIDs. Finally, female and male parental data sets in cross $\mathrm{BC}_{1}$ family, containing respectively 11.26 million filtered reads (comprising $2.25 \mathrm{~Gb}$ of data with a GC\% of 42.74) and 7.40 million filtered reads (comprising $1.48 \mathrm{~Gb}$ of data with a GC\% of 43.98), were correspondingly partitioned into 273667 and 248178 SLAF tags. And female and male parental

Table 1 Statistics of the genome survey from E. carinicauda

\begin{tabular}{ll}
\hline ltems & \\
\hline Genome size (Gb) & 9.33 \\
Heterozygosity & $0.26 \%$ \\
Repeat sequences & $76.62 \%$ \\
GC content & $40.01 \%$ \\
Data (Gb) & 294.46 \\
Depth $(X)$ & 31.57 \\
\hline
\end{tabular}

data sets in reverse cross $\mathrm{BC}_{1}$ family were correspondingly partitioned into 277696 and 251322 SLAF tags. From the 100 offspring of $\mathrm{BC}_{1}$ family, a total of 2.39 billion reads (with an average GC\% of 42.09) corresponding to $482,281.16 \mathrm{Mb}$ of data. For the 100 offspring of reverse cross $\mathrm{BC}_{1}$ family, a total of 2.21 billion reads (with an average GC\% of 42.30) corresponding to 449238.48 $\mathrm{Mb}$ of data.

\section{SNP marker genotyping and genetic linkage map}

After SLAF sequencing, 497888010 paired-end reads were generated for the mapping family (four parents and 200 progenies). A total of 301,314 polymorphic SLAF markers were identified from 1433342 SLAF markers, of which 10384 SNP markers could be successfully genotyped in both parents and offspring (Table 5). SNP

Table 2 Statistics of the genome assembly from E. carinicauda

\begin{tabular}{lll}
\hline Items & Contig & Scaffold \\
\hline Number & $31,371,476$ & $28,089,718$ \\
Total Length (bp) & $9,085,107,780$ & $9,185,133,106$ \\
$100-300$ & $6,721,269,034$ & $6,821,205,337$ \\
$300-500$ & $1,196,072,737$ & $1,002,403,357$ \\
$500-1000$ & $793,254,189$ & $809,021,501$ \\
$1000-2000$ & $297,655,772$ & $395,241,325$ \\
> 2000 & $76,855,759$ & $157,261,587$ \\
Longest & 135,963 & 135,963 \\
Shortest & 100 & 100 \\
Mean Length (bp) & 290 & 327 \\
N50 (bp) & 422 & 586 \\
N90 (bp) & 115 & 116 \\
\hline
\end{tabular}


Table 3 Pearson correlation coefficients ( $r$ ) for all pairwise combinations of the five growth-related traits $(P<0.001$ for all) in B backcross family

\begin{tabular}{llllll}
\hline Traits & $B L$ & $C L$ & $C W$ & $C H$ & $B W$ \\
\hline$B L$ & 1 & & & & \\
$C L$ & 0.946 & 1 & & & \\
$C W$ & 0.967 & 0.911 & 1 & & \\
$C H$ & 0.967 & 0.915 & 0.970 & 1 & \\
$B W$ & 0.975 & 0.924 & 0.962 & 0.970 & 1 \\
\hline
\end{tabular}

Note: $B L$ body length, $C L$ carpace length, $C W$ carpace width, $C H$ carpace height, $B W$ body weight, the same below

markers with the seven segregation patterns $(\mathrm{aa} \times \mathrm{bb}$, ab $\times \mathrm{cc}, \mathrm{cc} \times \mathrm{ab}, \mathrm{hk} \times \mathrm{hk}$, ef $\times \mathrm{eg}, \mathrm{lm} \times \mathrm{ll}, \mathrm{nn} \times \mathrm{np}$ ) could be used in linkage map construction for the $\mathrm{BC}_{1}$ family, of which aa $\times$ bb was one of the major pattern and most efficient markers for map construction (Fig. 2). The average read depth in backcross family of genotyped markers were $6.52,15.36$ and 22.75 in the offspring, male and female parents, respectively (Additional file 3: Table S3). And the average read depth in reverse backcross family of genotyped markers were 7.09, 16.90 and 28.51 in the offspring, male and female parents, respectively (Additional file 4: Table S4).

High-resolution SLAF-based SNP genetic maps of $E$. carinicauda based on backcross populations were first constructed using the pseudo-testcross strategy. The $\mathrm{BC}_{1}$ family genetic linkage map contained 45 linkage groups with 4050 markers and the reverse cross $\mathrm{BC}_{1}$ family genetic linkage map contained 45 linkage groups with 6,334 markers (Table 6, Fig. 3, Fig. 4, Additional file 5: Table S5 and Additional file 6: Table S6). The total map distance of two maps were $2939.27 \mathrm{cM}$ with an average inter-locus distance of $0.73 \mathrm{cM}$ and $3460.28 \mathrm{cM}$ with an average interlocus distance of $0.55 \mathrm{cM}$ respectively, which covered 97.65 and $98.44 \%$ respectively of the genome based on the total length of the genome.

\section{Genetic linkage map integration}

Among the 4050 markers in the backcross map, 2733 markers could align to 1468 genomic scaffolds/contigs with high confidence (Additional file 7: Table S7). And

Table 4 Pearson correlation coefficients $(r)$ for all pairwise combinations of the five growth-related traits $(P<0.001$ for all) in $B^{\prime}$ reverse backcross family

\begin{tabular}{llllll}
\hline Traits & $B L$ & $C L$ & $C W$ & $C H$ & $B W$ \\
\hline$B L$ & 1 & & & & \\
$C L$ & 0.919 & 1 & & & \\
$C W$ & 0.837 & 0.774 & 1 & & \\
$C H$ & 0.934 & 0.913 & 0.816 & 1 & \\
$B W$ & 0.983 & 0.920 & 0.823 & 0.925 & 1 \\
\hline
\end{tabular}

Table 5 Statistics of SLAF and SNP data

\begin{tabular}{lll}
\hline Items & Backcross family & $\begin{array}{l}\text { Reverse } \\
\text { Backcross family }\end{array}$ \\
\hline Number of reads & $257,646,490$ & $240,241,520$ \\
Number of SLAF & 835,181 & 598,161 \\
Polymorphic SLAF & 164,444 & 136,870 \\
Polymorphic SNP & 4050 & 6334 \\
$\begin{array}{l}\text { Average depth in } \\
\text { male parent }\end{array}$ & 15.36 & 16.9 \\
$\begin{array}{l}\text { Average depth in } \\
\text { female parent }\end{array}$ & 22.75 & 28.51 \\
$\begin{array}{l}\text { Average depth in } \\
\text { offspring }\end{array}$ & 6.52 & 7.09 \\
\hline
\end{tabular}

91 markers were anchored to unigenes of transcriptome (Additional file 8: Table S8). In the reverse backcross map, 4,297 markers could align to 2403 genomic scaffolds/contigs with high confidence (Additional file 9: Table S9). And 156 markers were anchored to unigenes of transcriptome (Additional file 10: Table S10). Based on blast information, there were some markers of genetic linkage maps, genomic scaffolds/contigs and unigenes of transcriptome that can be integrated together (Fig. 5, Fig. 6). 7,030 markers could be aligned to the genomic scaffolds/contigs or transcriptome unigenes, of which 846 markers could be blasted via with the public databases.

\section{QTL mapping of growth-related traits}

The high-resolution genetic linkage map was used in the present study for QTL mapping of growth-related traits using MapQTL 4.0 software. In the map of Backcross family, 7 QTLs were detected for all the five growth-related traits, which were distributed on LG1, LG2 and LG20 (Table 7 and Fig. 7). These QTLs with LOD values of 2.66-3.62 contributed to PVE of $11.5-15.7 \%$. Among them, $q B W-1$ located at $49.652-79.283 \mathrm{cM}$ of LG2 with the highest LOD score of 3.62, and correspondingly had the higher PVE value of $15.4 \%$. Some QTL intervals were clustered together on the same respective linkage groups (LGs). One major cluster containing three QTLs $(q B L-2$, $q C H-1$ and $q B W-1$ ) was detected between the positions of 47.024-79.283 cM on LG2. On LG20, another cluster located within the region $(0-1.826 \mathrm{cM})$ also consisted of two QTLs $(q C W-1$ and $q B W-2)$. A total of 16 markers were located in the QTL intervals, among which, 8 markers (50\%) distributed on LG2. In the map of reverse Backcross family, a total of 16 QTLs were detected for all the five growth-related traits, which were distributed on LG1, LG10, LG13, LG17, LG19, LG24 and LG38 (Table 7 and Fig. 8). The QTLs with LOD values of 2.02.74 contributed to $P V E$ of $8.8-11.9 \%$. Of which, one major cluster containing four QTLs $(q B L-2, q C W-3$, 


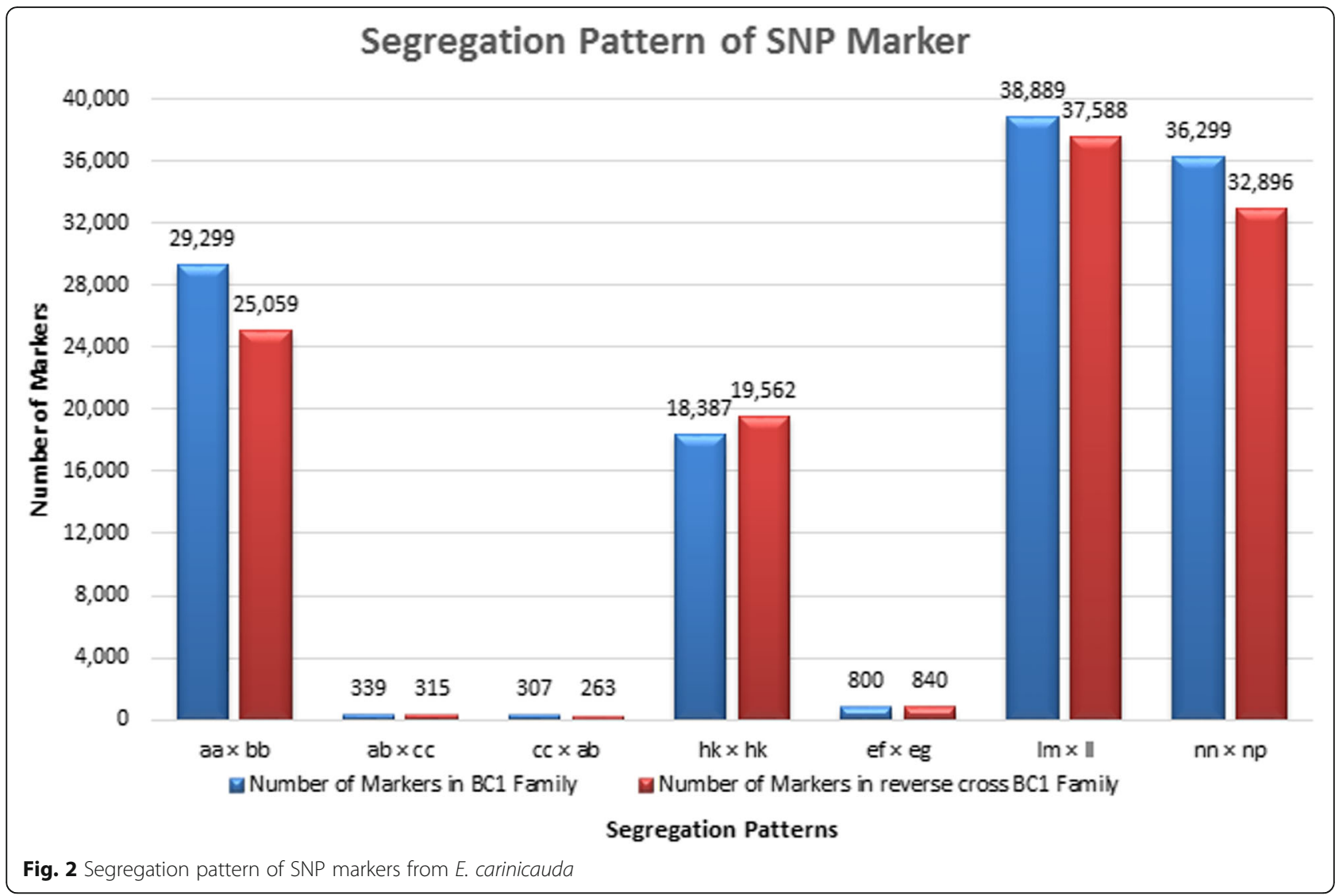

$q C H-2$ and $q B(W-4)$ was detected at the positions of $72.613 \mathrm{cM}$ on LG19. Another cluster situated within the region $(43.872-60.091 \mathrm{cM})$ on LG19 also consisted of four QTLs $(q B L-1, q C W-2, q C H-1$ and $q B W-3)$. A total of 25 markers were located in the QTL intervals, among which, 10 markers (40.0\%) distributed on LG10.

\section{Discussion}

E. carinicauda is an economic marine shrimp species in eastern China. Given its economic importance, great researches have been made to investigate the genomic characteristics of this species. In the present study, a total of $294.46 \mathrm{~Gb}$ high-quality reads were obtained, covering approximately 31.57 -fold genome size of $E$. carinicauda. The genome size of E. carinicauda was estimated to $9.33 \mathrm{~Gb}$ based on the K-mer analysis, which is relatively larger than the previous results and many commonly recognized decapod shrimps [30]. The genome size of $E$. carinicauda is higher than those of penaeid shrimps [20,31], caridean shrimp Neocaridina denticulate [32], Eriocheir sinensis [33] and Portunus trituberculatus [34] by 4.0-, 3.1-, 8.2- and 11.3-folds, respectively. The genome survey analysis revealed the complexity of the E. carinicauda genome. Approximately $76.62 \%$ of the genome was occupied by repetitive sequences, which was very similar to the $L$. vannamei genome [20] and the $N$. denticulate genome [32]. A total of 31371476 contigs with N50 length of $422 \mathrm{bp}$ was obtained, which was higher than that of previous study [30]. And a total of 65772 unigenes with mean length of 1386 bp were obtained, which was relatively smaller than those of decapod shrimps [30].

The reciprocal-cross backcross population of $E$. carinicauda were firstly obtained for construction of genetic linkage map and the growth traits were analyzed. Significant correlations among five growth traits (BL, BW, CL, $\mathrm{CW}$ and $\mathrm{CH}$ ) have been reported in other species, such as Atlantic salmon [35] and Zhikong Scallop [36]. Similarly with our study, five growth traits were closely related to each other, with the highest correlation coefficient $(r=0.96)$ between BL and BW, and relatively low correlation coefficient $(r=0.81)$ between $\mathrm{CW}$ and other traits.

High-resolution genetic linkage map is an essential tool for research of genetics and genomics, such as comparative genome analysis and QTL fine mapping [37]. SNPs are especially suitable for genetic linkage map construction, which representing the most abundant and stable form of genetic variation in most genomes [38]. In this study, 301314 polymorphic SLAF tags were developed from 4 E. carinicauda parents and 200 offspring individuals. 4050 SNPs distributed throughout the 
Table 6 Characteristics of genetic maps of E. carinicauda Backcross families

\begin{tabular}{|c|c|c|c|c|c|c|}
\hline \multirow[t]{2}{*}{ LG_ID } & \multicolumn{3}{|c|}{ Map of Backcross family } & \multicolumn{3}{|c|}{ Map of Reverse Backcross family } \\
\hline & Total marker & $\begin{array}{l}\text { Total } \\
\text { Distance(cM) }\end{array}$ & $\begin{array}{l}\text { Average } \\
\text { Distance(cM) }\end{array}$ & Total marker & Total Distance(cM) & Average Distance(cM) \\
\hline 1 & 61 & 64.70 & 1.08 & 134 & 97.93 & 0.74 \\
\hline 2 & 65 & 79.28 & 1.24 & 61 & 50.22 & 0.84 \\
\hline 3 & 49 & 35.89 & 0.75 & 64 & 41.62 & 0.66 \\
\hline 4 & 152 & 78.00 & 0.52 & 147 & 91.59 & 0.63 \\
\hline 5 & 203 & 112.36 & 0.56 & 326 & 90.52 & 0.28 \\
\hline 6 & 36 & 20.73 & 0.59 & 92 & 81.75 & 0.90 \\
\hline 7 & 149 & 92.27 & 0.62 & 71 & 43.80 & 0.63 \\
\hline 8 & 263 & 156.72 & 0.60 & 128 & 33.88 & 0.27 \\
\hline 9 & 159 & 134.13 & 0.85 & 66 & 50.11 & 0.77 \\
\hline 10 & 65 & 44.18 & 0.69 & 62 & 57.45 & 0.94 \\
\hline 11 & 78 & 50.37 & 0.65 & 66 & 31.75 & 0.49 \\
\hline 12 & 116 & 64.73 & 0.56 & 99 & 46.28 & 0.47 \\
\hline 13 & 61 & 73.24 & 1.22 & 156 & 68.49 & 0.44 \\
\hline 14 & 80 & 72.07 & 0.91 & 125 & 75.86 & 0.61 \\
\hline 15 & 65 & 42.98 & 0.67 & 120 & 62.85 & 0.53 \\
\hline 16 & 42 & 14.39 & 0.35 & 103 & 67.73 & 0.66 \\
\hline 17 & 34 & 21.80 & 0.66 & 326 & 123.02 & 0.38 \\
\hline 18 & 34 & 53.71 & 1.63 & 89 & 41.19 & 0.47 \\
\hline 19 & 56 & 55.01 & 1.00 & 199 & 111.98 & 0.57 \\
\hline 20 & 111 & 45.13 & 0.41 & 79 & 62.46 & 0.80 \\
\hline 21 & 124 & 95.42 & 0.78 & 129 & 81.59 & 0.64 \\
\hline 22 & 86 & 51.35 & 0.60 & 86 & 84.83 & 1.00 \\
\hline 23 & 123 & 80.31 & 0.66 & 128 & 65.67 & 0.52 \\
\hline 24 & 156 & 127.80 & 0.82 & 133 & 99.18 & 0.75 \\
\hline 25 & 39 & 40.86 & 1.08 & 159 & 84.33 & 0.53 \\
\hline 26 & 55 & 46.57 & 0.86 & 175 & 118.87 & 0.68 \\
\hline 27 & 201 & 65.40 & 0.33 & 183 & 99.70 & 0.55 \\
\hline 28 & 105 & 57.82 & 0.56 & 66 & 38.16 & 0.59 \\
\hline 29 & 145 & 134.27 & 0.93 & 73 & 37.02 & 0.51 \\
\hline 30 & 106 & 90.32 & 0.86 & 166 & 69.72 & 0.42 \\
\hline 31 & 200 & 133.71 & 0.67 & 125 & 53.11 & 0.43 \\
\hline 32 & 108 & 85.05 & 0.79 & 259 & 119.91 & 0.46 \\
\hline 33 & 38 & 37.02 & 1.00 & 98 & 109.44 & 1.13 \\
\hline 34 & 50 & 49.18 & 1.00 & 166 & 83.23 & 0.50 \\
\hline 35 & 35 & 11.40 & 0.34 & 81 & 48.79 & 0.61 \\
\hline 36 & 90 & 83.25 & 0.94 & 101 & 51.73 & 0.52 \\
\hline 37 & 68 & 69.00 & 1.03 & 206 & 117.64 & 0.57 \\
\hline 38 & 54 & 50.17 & 0.95 & 106 & 76.79 & 0.73 \\
\hline 39 & 84 & 67.86 & 0.82 & 193 & 50.52 & 0.26 \\
\hline 40 & 38 & 28.04 & 0.76 & 437 & 133.99 & 0.31 \\
\hline 41 & 32 & 11.85 & 0.38 & 124 & 104.72 & 0.85 \\
\hline 42 & 63 & 54.44 & 0.88 & 163 & 108.97 & 0.67 \\
\hline
\end{tabular}


Table 6 Characteristics of genetic maps of E. carinicauda Backcross families (Continued)

\begin{tabular}{|c|c|c|c|c|c|c|}
\hline \multirow[t]{2}{*}{$\overline{L G \_I D}$} & \multicolumn{3}{|c|}{ Map of Backcross family } & \multicolumn{3}{|c|}{ Map of Reverse Backcross family } \\
\hline & Total marker & $\begin{array}{l}\text { Total } \\
\text { Distance(cM) }\end{array}$ & $\begin{array}{l}\text { Average } \\
\text { Distance(cM) }\end{array}$ & Total marker & Total Distance(cM) & Average Distance(cM) \\
\hline 43 & 50 & 67.97 & 1.39 & 211 & 106.13 & 0.51 \\
\hline 44 & 32 & 18.70 & 0.60 & 176 & 101.18 & 0.58 \\
\hline 45 & 89 & 69.82 & 0.79 & 77 & 84.58 & 1.11 \\
\hline Total & 4,050 & $2,939.27$ & 0.73 & 6,334 & $3,460.28$ & 0.55 \\
\hline Average & 90 & 65.32 & 0.73 & 140.75 & 76.90 & 0.55 \\
\hline Genome coverage (\%) & & $97.65 \%$ & & & $98.44 \%$ & \\
\hline
\end{tabular}

backcross genetic map and 6334 SNPs distributed throughout the reverse backcross genetic map, providing a large number of genomic and genetic resources for $E$. carinicauda. We successfully constructed the first highresolution genetic linkage map with 45 linkage groups, which was in accordance with karyotypes of $E$. carinicauda. The total length of the backcross map was $2939.27 \mathrm{cM}$ with an average distance between adjacent markers of $0.73 \mathrm{cM}$, and the total length of the reverse backcross map was $3460.28 \mathrm{cM}$ with an average distance between adjacent markers of $0.55 \mathrm{cM}$. The mapping resolution of reciprocal backcross population exceeded those of P. monodon (0.9 cM) [19], and similar to L. vannamei $(0.7 \mathrm{cM})$ [20], P. trituberculatus $(0.51 \mathrm{cM})$ [34] and E. sinensis $(0.49 \mathrm{cM})$ [22]. Additionally, the marker developed in our study contained a $200 \mathrm{bp}$ terminal sequences, which provided enough information for trait-related QTLs verfication and comparative genome analysis.
To identify potential growth-related genes, we compared the detected QTLs with the scaffold assembly from genome survey and transcriptome of E. carinicauda [39]. Based on the integrated map, 91 markers could be aligned to genome assemblies or transcript sequences in the backcross map, and 13 of 91 markers could annotated candidate genes via blast in public databases. Meanwhile, 156 markers could be aligned to genome assemblies or transcript sequences in the reverse backcross map, and 25 of 156 markers could annotated candidate genes via blast in public databases. These aligned genes were related to many important physiological processes and functions, such as protein kinase activity [40], regulation of apoptotic process [41] and sequence-specific DNA binding transcription factor activity [42]. These annotated genes provide candidate information for analysis of economic traits in $E$. carinicauda.

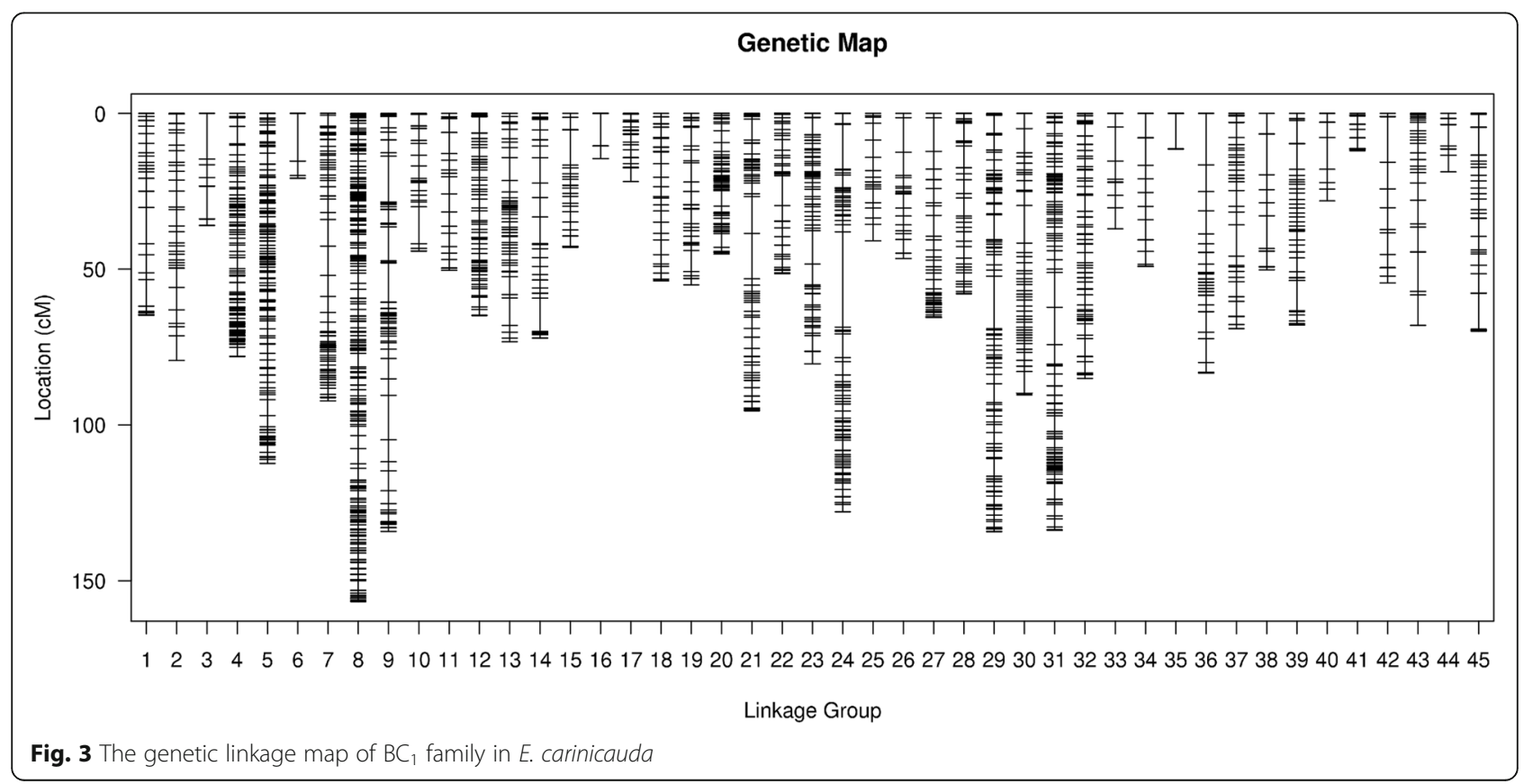




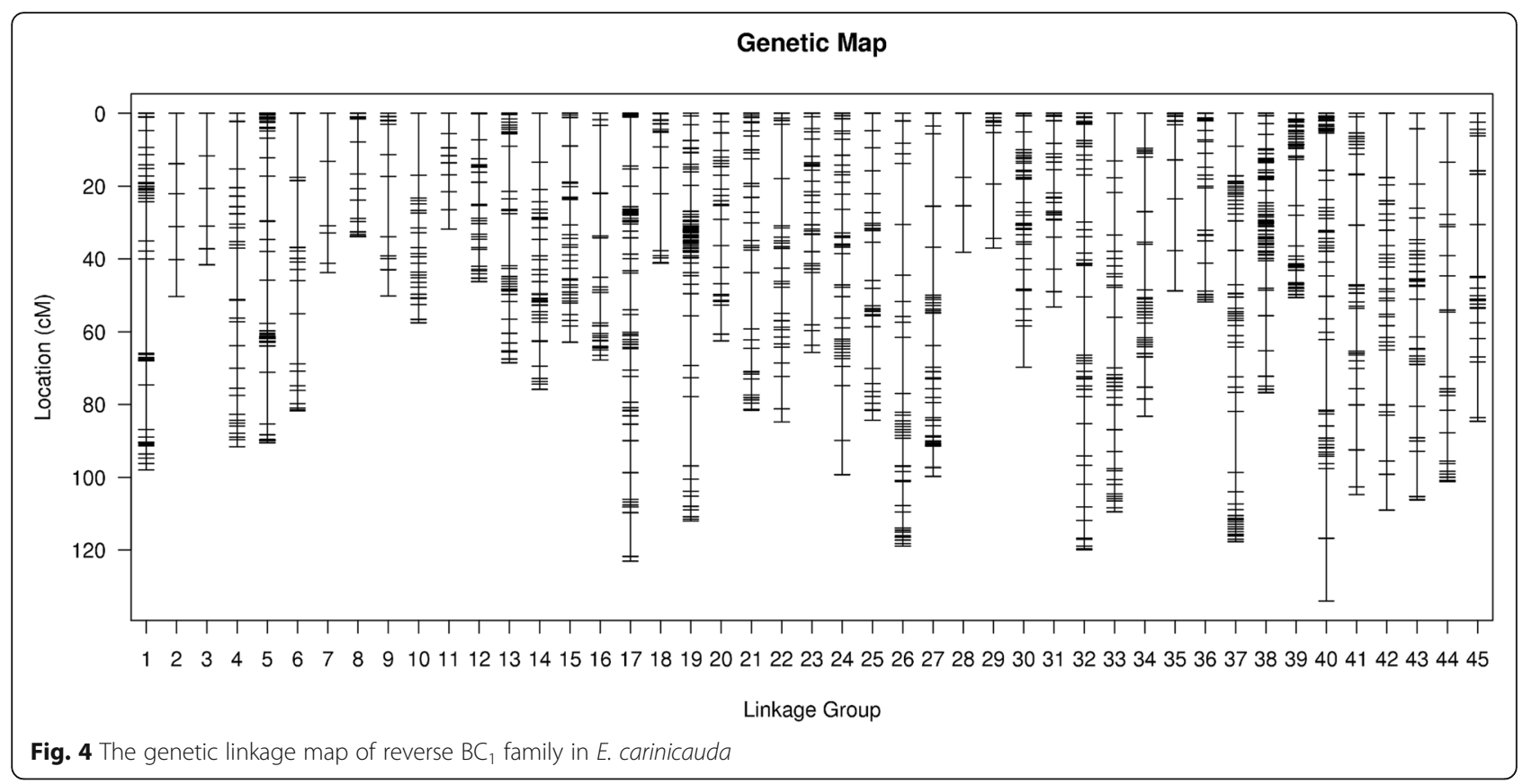

QTL mapping is a very effective strategy to locate traitrelated genes for MAS in genetic breeding of aquaculture species [36]. The high-resolution genetic map constructed in this study provided important tools for performing QTL fine mapping for economical traits of $E$. carinicauda. In the present study, 7 QTLs associated with growth traits were found to be distributed on three LGs (LG1, LG2 and LG20) in the backcross linkage map. And 16 QTLs associated with growth traits were found to be distributed on six LGs (LG1, LG10, LG13, LG17, LG19, LG24 and LG38) in the reverse backcross linkage map. Interestingly, most of the QTLs were concentrated within a narrow region (cluster) on the LGs. In the backcross linkage map, three QTLs were clustered together (47.024-79.283 cM) on LG2 corresponding to assembly contig85361652 $(2.2 \mathrm{~kb})$ and scaffold1051673 $(6.4 \mathrm{~kb})$. And two QTLs were found in another cluster (0$1.826 \mathrm{cM})$ on LG20, corresponding to scaffold1658074 (1.8 $\mathrm{kb})$. Meanwhile, in the reverse backcross map, three QTLs were clustered together $(60.091-60.576 \mathrm{cM})$ on LG17 corresponding to assembly contig84295559 (1.3 kb), scaffold234386 (1.2 kb) and scaffold037331 (3.7 kb). Additionally, three QTLs were also clustered together (90.716$91.096 \mathrm{cM}$ ) on LG19, indicating that the growth traits may be controlled by the same genes. The quite small genetic and physical distances among QTLs in specific clusters suggested that the individual clusters might be highly effective QTLs. The results were in accordance with the previous results in P. trituberculatus [34]. The demonstration of one trait controlled by a few significant QTLs with higher PVE values was consistent with the characteristics of growth traits controlled by several major genes with higher heritability [43, 44].

\section{Conclusions}

In conclusion, genome size of E. carinicauda was estimated as $9.33 \mathrm{~Gb}$ with remarkably low heterozygosity. Large-scale SNPs identified and genotyped via SLAF-seq technology were used to construct a high-resolution genetic map of the ridgetail white prawn. The developed genetic map is the most comprehensive genetic map to date for this species. Based on SNP mapping analysis, we identified 23 positive QTLs for growth traits that will be helpful to clarifying the genetic mechanism of growth regulation in the ridgetail white prawn. The obtained SNPs and high-resolution linkage map, coupled with genome survey and transcriptome established an important platform for QTL mapping and also provided an extremely useful resource for future molecular breeding efforts such as genome selection.

\section{Methods}

\section{Genome survey analysis}

DNA samples of E. carinicauda was extracted from the muscles of parent of backcross family for sequencing. Six paired-end DNA libraries with an insert size of $270 \mathrm{bp}$ were constructed following the Illumina operating protocols. The paired-end sequencing was performed on the Illumina Hiseq 4000 platform (Illumina, Inc.; San Diego, CA, USA). The raw data were trimmed to filter out lowquality reads and adapter contaminates using NGS QC Toolkit [45]. De novo assembly was performed to obtain contigs using SOAP denovo software [46] (https://sourceforge.net/projects/soapdenovo2/files/SOAPdenovo2/) with the following parameters: the $\mathrm{k}$ value in $\mathrm{K}$-mer was set at 23, unsolve repeats by reads fill gaps in scaffolds. 


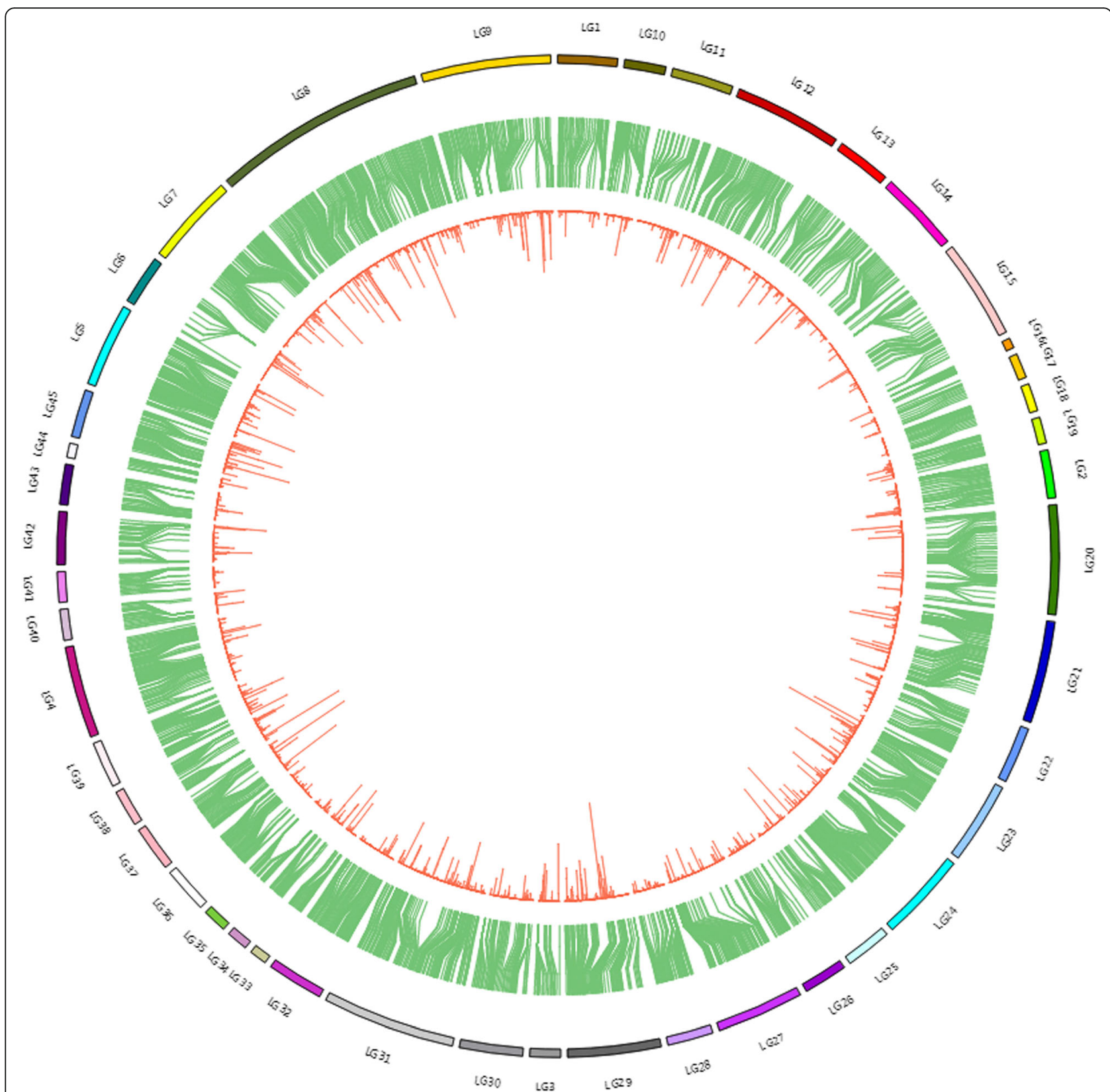

Fig. 5 The integrated map of genome, $\mathrm{BC}_{1}$ map and transcriptome in E. carinicauda. Outer ring, the linkage group; Intermediate ring, contigs or scaffolds of genome assembly aligned with markers from the linkage map; Inner ring, unigene sequences of transcriptome aligned with scaffold/contig sequences

\section{Mapping population and phenotype data}

The reciprocal-cross backcross families of E. carinicauda were constructed in the breeding center of Yellow Sea Fisheries Research Institute, CAFS. Firstly, A $F_{1}$ full-sib family was created from male parent prawn from Rizhao population and female prawn from Xiangshan population. Then, cross $\mathrm{BC}_{1}$ family was obtained from the female $\mathrm{F}_{1}$ individuals and male parent, and reverse cross $\mathrm{BC}_{1}$ family come from the male $F_{1}$ individuals and female parent. Finally, two reciprocal-cross backcross families were obtained after 3 months of growth. The parents prawn and $\mathrm{BC}_{1}$ progenies were raised in a $200 \mathrm{~L}$ and $500 \mathrm{~L}$ tanks respectively and fed four times daily under standard feeding management (5$10 \%$ feed/body weight ratio daily). The oxygen level in sea water was maintained at $5 \mathrm{mg} / \mathrm{L}$ or above. Growth-related traits including body weight (BW), body length (BL), carapace length $(\mathrm{CL})$, width $(\mathrm{CW})$ and height $(\mathrm{CH})$ of 200 $\mathrm{BC}_{1}$ progenies randomly selected after 3 months of growth were measured for each individual. Statistical analysis of the growth traits data was conducted with SPSS 17.0.

DNA samples were extracted from the muscles of the parental prawns and $200 \mathrm{BC}_{1}$ progenies using traditional 


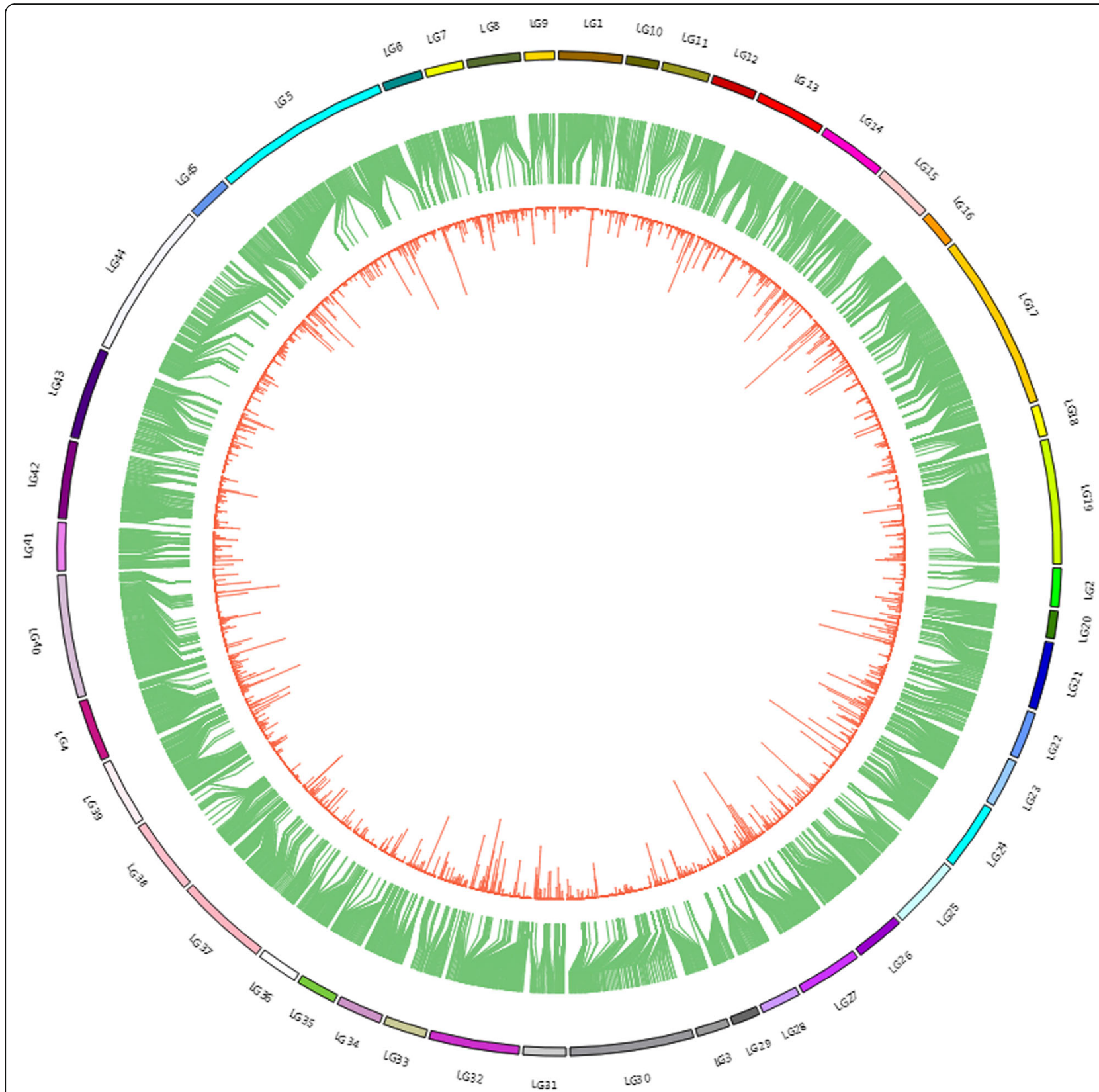

Fig. 6 The integrated map of genome, reverse $\mathrm{BC}_{1}$ map and transcriptome in E. carinicauda. Outer ring, the linkage group; Intermediate ring, contigs or scaffolds of genome assembly aligned with markers from the linkage map; Inner ring, unigene sequences of transcriptome aligned with scaffold/contig sequences

phenol-chloroform extraction in combination with RNase treatment. Before construction of SLAF-seq libraries, all DNA samples were quantified using a NanoDrop 1000 spectrophotometer (Thermo Scientific, Wilmington, DE, USA), and the concentrations were adjusted to $50 \mathrm{ng} / \mu \mathrm{L}$.

\section{SLAF library construction and high-throughput sequencing}

Based on the size of E. carinicauda genome, content of $\mathrm{GC}$ and in-silico analysis, endonuclease Hae III were used to digest the genome. SLAF library were constructed as described by Sun et al. [23] with minor modifications. For the $\mathrm{BC}_{1}$ population, endonuclease Hae III (New England Biolabs, NEB, USA) were chosen to digest the genomic DNA of E. carinicauda. Subsequently, a single nucleotide (A) overhang was attached to the digested fragments by using Klenow Fragment $\left(3^{\prime} \rightarrow 5^{\prime}\right.$ exo ${ }^{-}$) (NEB, USA) and dATP at $37^{\circ} \mathrm{C}$. PAGE-purified duplex tag-labelled sequencing adapters (Life Technologies, USA) were then ligated to the A-tailed fragments 
Table 7 Characteristics of growth related QTLs

\begin{tabular}{|c|c|c|c|c|c|c|c|c|}
\hline Maps & Traits & QTL & Linkage Group & Start (cM) & End (cM) & Marker Number & Max LOD & Max PVE \\
\hline \multirow[t]{7}{*}{ Backcross map } & $B L$ & $q B L-1$ & 2 & 47.024 & 47.024 & 1 & 3.09 & 13.3 \\
\hline & & $q B L-2$ & 2 & 79.283 & 79.283 & 1 & 3.00 & 12.9 \\
\hline & $C L$ & $q C L-1$ & 1 & 12.586 & 12.586 & 1 & 2.66 & 11.5 \\
\hline & $C W$ & $q C W-1$ & 20 & 0 & 1.826 & 7 & 3.57 & 15.7 \\
\hline & $\mathrm{CH}$ & $\mathrm{qCH}-1$ & 2 & 47.024 & 79.283 & 3 & 3.27 & 14.0 \\
\hline & $B W$ & $q B W-1$ & 2 & 49.652 & 79.283 & 8 & 3.62 & 15.4 \\
\hline & & $q B W-2$ & 20 & 0 & 0 & 2 & 3.26 & 14.3 \\
\hline \multirow[t]{16}{*}{ Reverse backcross map } & $B L$ & $q B L-1$ & 17 & 43.872 & 60.091 & 2 & 2.36 & 10.3 \\
\hline & & $q B L-2$ & 19 & 72.613 & 72.613 & 1 & 2.74 & 11.9 \\
\hline & $C L$ & $q C L-1$ & 1 & 90.716 & 90.716 & 1 & 2.12 & 9.3 \\
\hline & & $\mathrm{qCl}-2$ & 13 & 21.418 & 21.418 & 1 & 2.04 & 9.0 \\
\hline & & $q C L-3$ & 24 & 35.908 & 36.779 & 4 & 2.24 & 9.8 \\
\hline & $C W$ & $q C W-1$ & 1 & 90.716 & 91.096 & 2 & 2.12 & 9.3 \\
\hline & & $q C W-2$ & 17 & 43.872 & 60.995 & 7 & 2.38 & 10.4 \\
\hline & & $q C W-3$ & 19 & 72.613 & 72.613 & 1 & 2.0 & 8.8 \\
\hline & $\mathrm{CH}$ & $\mathrm{qCH}-1$ & 17 & 43.872 & 60.091 & 2 & 2.15 & 9.4 \\
\hline & & $q \mathrm{CH}-2$ & 19 & 72.613 & 72.613 & 1 & 2.26 & 9.9 \\
\hline & & $q \mathrm{CH}-3$ & 24 & 36.779 & 36.779 & 2 & 2.05 & 9.0 \\
\hline & & $q \mathrm{CH}-4$ & 38 & 13.293 & 13.293 & 1 & 2.14 & 9.4 \\
\hline & $B W$ & $q B W-1$ & 1 & 90.716 & 91.096 & 2 & 2.09 & 9.2 \\
\hline & & $q B W-2$ & 10 & 57.446 & 57.447 & 10 & 2.03 & 8.9 \\
\hline & & $q B W-3$ & 17 & 43.872 & 60.091 & 2 & 2.39 & 10.4 \\
\hline & & $q B W-4$ & 19 & 72.613 & 72.613 & 1 & 2.49 & 10.8 \\
\hline
\end{tabular}

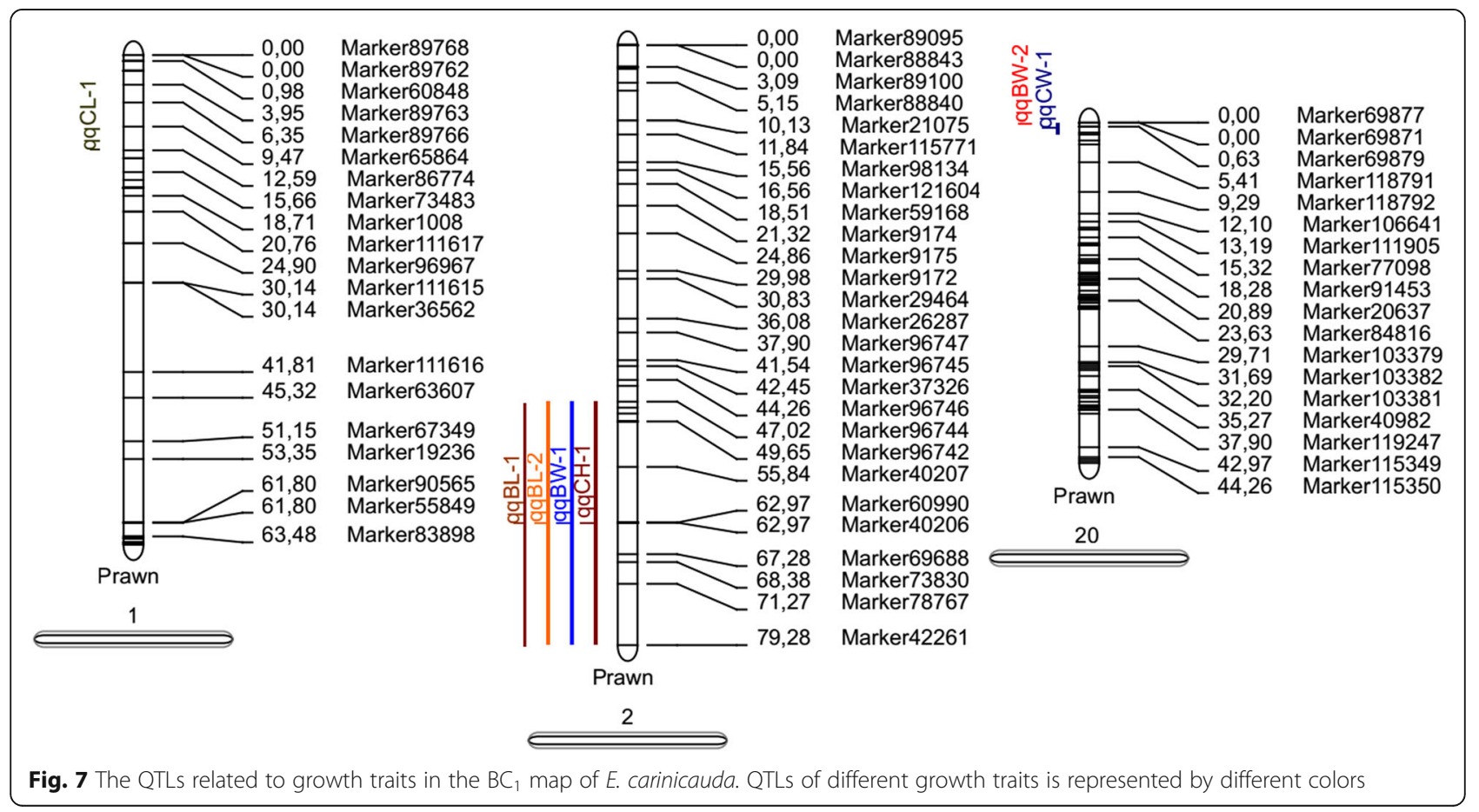




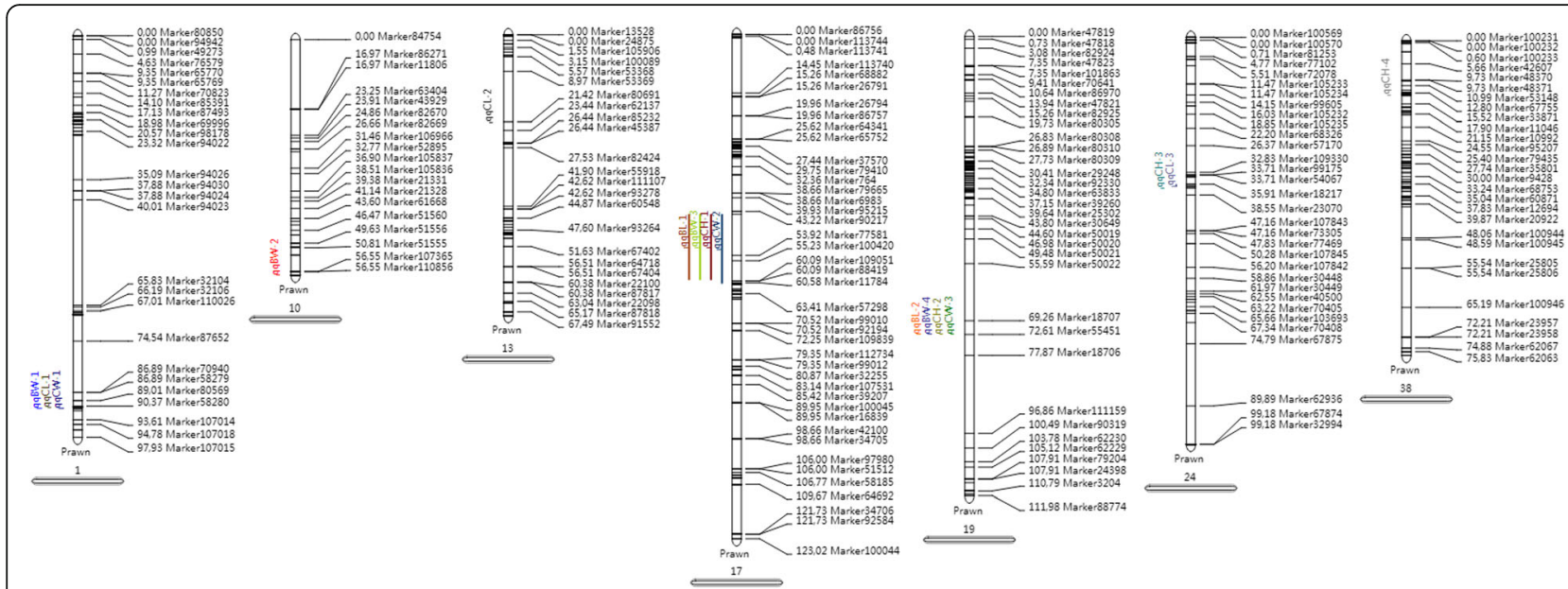

Fig. 8 The QTLS related to growth traits in the reverse $B C_{1}$ map of E. carinicauda. QTLS of different growth traits is represented by different colors

using $\mathrm{T}_{4}$ DNA ligase. PCR was performed using diluted restriction-ligation DNA samples, dNTP, Q5 High-Fidelity DNA Polymerase and PCR primers (Forward primer: 5'-AATGATACGGCGACCACCGA-3', reverse primer: 5' -CAAGCAGAAGACGGCATACG-3') (PAGEpurified, Life Technologies, USA). The PCR products were purified by using Agencourt AMPure XP beads (Beckman Coulter, High Wycombe, UK) and then pooled. The pooled samples were separated using $2 \%$ agarose gel electrophoresis. The fragments of 364 to $444 \mathrm{bp}$ (with indexes and adaptors) in size were excised and purified using a QIAquick Gel Extraction Kit (Qiagen, Hilden, Germany). The gel-purified products were then diluted and pair-end sequenced (Each end $125 \mathrm{bp}$ ) on Illumina HiSeq 2500 system (Illumina, Inc.; San Diego, CA, USA) according to the manufacturer's protocols. To avoid false positive reads, the sequence error rate was estimated using the data of Oryza sativa as a control. The ratio of high quality reads with quality scores greater than Q30 (indicating a 1\% chance of an error, and thus $99 \%$ confidence) in the raw reads and $\mathrm{GC}$ amounts were calculated for quality control.

\section{SNP calling and genotyping}

After filtering out the low-quality reads (quality score < $30 e$ ), the remaining reads were sorted to each progeny according to duplex barcode sequences. Then each of the high-quality read was trimmed off 5-bp terminal position, sequences clustered by similarity above $90 \%$ were defined as one SLAF locus [47]. Genome Analysis Toolkit (GATK) [48] and Sequence Alignment/Map tools (SAMtools) [43] was used for calling the SNPs. Local realignment was performed to avoid false alignments near InDels. The "Unified Genotyper" module of GATK was used for variant calling. Both SAMtools and GATK tools were used to identify SNPs, and their intersection was merged as the candidate SNP dataset. Only biallelic SNPs were identified as the final SNP dataset. Polymorphic SNP markers were converted to four segregation patterns $(\mathrm{hk} \times \mathrm{hk}, \mathrm{lm} \times \mathrm{ll}, \mathrm{nn} \times \mathrm{np}$ and aa $\times$ bb). The reciprocal-cross backcross families of E. carinicauda were obtained from the crosses between parents and $F_{1}$ individuals with genotype aa or bb. Thus, only the SNPs whose segregation patterns were aa $\times$ bb were used to construct the genetic linkage map. The average sequencing depths of SNPs were more than 18-fold in the parents and greater than 6 -fold in the progeny, respectively. And a progeny contained more than $70 \%$ of the SNPs in both parents, i.e., 70\% integrity of SNPs in individuals. A $\chi^{2}$ test was implemented for each SNP with a null hypothesis that the two alleles at a locus segregated with 1:1 ratio in our BC population. All SNPs that significantly deviated from this ratio $(P<0.001)$ were eliminited from the SNP dataset.

\section{Genetic linkage map construction}

To ensure the quality of the genetic map, HighMap strategy was utilized to sort the SNP markers and correct genotyping errors within LGs [44]. Marker loci were distributed primarily into LGs by the modified logarithm of odds (MLOD) scores $>5.0$ and a maximum recombination fraction of 0.4. MSTmap algorithms [49] and SMOOTH algorithms [50] were chosen to order the SNP markers and correct genotyping errors, respectively. The LGs were constructed as follows: Primary marker orders were first obtained by their location on the chromosomes according to the relationship of the ordered markers, and genotyping errors or deletions were corrected using the SMOOTH algorithm; then, MSTmap was used to sort the map and SMOOTH was reused to correct the newly ordered genotypes. The processes were conducted repetitively to ensure the accuracy of marker 
order and map distances, finally high-quality maps were obtained after four or more processes.

\section{Integration of genetic linkage map, genome and transcriptome}

The reference transcriptome unigenes were de novo assembled using the obtained reads from our previous work [39]. The clean reads were assembled with Trinity program as reported previously [51], followed by TIGR Gene Indices Clustering Tools (TGICL) [52]. The reads were then mapped back to contigs with paired-end reads to detect contigs from the same transcript and the distances between these contigs. The letter $\mathrm{N}$ was used to connect each two contigs to represent unknown sequences, and then for Scaffold. Finally, sequences were obtained that lacked $\mathrm{N}$ and could not be extended on either end [53], and were defined as unigenes. These unigenes were aligned by BlastX to public databases including the NCBI, the Swiss-Prot database, the KEGG database, and the COG database (E-value $\leq 1.0 \times 10^{-5}$ ).

An integrated map was constructed using the map markers, transcriptome unigenes and genomic scaffolds/ contigs mainly via BLAT and the Circos tool [54]. Before BLAT analyzing, the Repeat-Masker software was run to increase the accuracy of alignment by masking homologous repeats [55]. Three sets of data were integrated and aligned to each other by BLAT analysis. The outer ring of the integration map represented the physical distance of each LG, which was the total length of genomic scaffold/contig alignment to the LG with the default parameters (minMatch 2, minScore 30, minIdentity 90, maxGap 2). The middle ring represented the alignment relation between SNP markers and genomic scaffold/ contig. If the two ends of a marker matched different scaffold/contig sequences, then the marker was linked to the two scaffold/contig sequences. The inner ring represented the alignment relation between transcriptome unigenes and genome scaffold/contig sequences, and calculated the number of unigenes per $\mathrm{kb}$ of genome sequence.

\section{QTL analysis for growth-related traits}

The growth traits phenotypic data of backcross population were shown in Additional file 1: Table S1. QTL mapping analysis were conducted using MapQTL 4.0 software by the composite interval mapping (CIM) method [27, 56]. CIM method was used with a walking speed of $1 \mathrm{cM}$. Two-LOD support intervals were constructed with $95 \%$ confidence intervals [57]. The significance of each QTL interval was verified using likelihood-ratio statistic (LOD). The threshold of the LOD score for significance $(P=0.05)$ was detected using 1000 permutations. Calculation of the percentage of growth-traits variation explained $(P V E)$ by each QTL
(Expl. \%) was conducted with MapQTL 4.0 based on the variation within the mapping population.

\section{Additional files}

Additional file 1: Table S1. Phenotypic data of sequenced individuals from $\mathrm{BC}_{1}$ and reverse $\mathrm{BC}_{1}$ families of E. carinicauda. (XLSX $24 \mathrm{~kb}$ )

Additional file 2: Table S2. The predicted genes from the genome survey of E. carinicauda. (XLSX $9232 \mathrm{~kb}$ )

Additional file 3: Table S3. The SNP marker number and the read depth of each genotyped marker in the backcross mapping family. (XLSX $15 \mathrm{~kb})$

Additional file 4: Table S4. The SNP marker number and the read depth of each genotyped marker in the reverse backcross mapping family. (XLSX $15 \mathrm{~kb}$ )

Additional file 5: Table S5. Marker information of Backcross linkage map. (XLSX 107 kb)

Additional file 6: Table S6. Marker information of reverse Backcross linkage map. (XLSX $151 \mathrm{~kb}$ )

Additional file 7: Table S7. Markers of Backcross linkage map anchored to scaffolds. (XLSX $151 \mathrm{~kb}$ )

Additional file 8: Table S8. Markers of Backcross linkage map anchored to transcriptome. (XLSX $28 \mathrm{~kb}$ )

Additional file 9: Table S9. Markers of reverse Backcross linkage map anchored to scaffolds. (XLSX $227 \mathrm{~kb}$ )

Additional file 10: Table S10. Markers of reverse Backcross linkage map anchored to transcriptome. (XLSX $39 \mathrm{~kb}$ )

\section{Abbreviations \\ BC: Backcross; LGs: Linkage Groups; LOD: Logarithm of Odds; MAS: Marker- Assisted Selection; NGS: Next Generation Sequencing; PVE: Phenotypic Variance Explained; QTL: Quantitative Trait Loci; SLAF: Specific-Locus Amplified Fragment sequencing; SNP: Single Nucleotide Polymorphism}

\section{Acknowledgements}

Not applicable.

\section{Authors' contributions}

JTL designed and guided this study. JL proposed the conception of this study and constructed the Backcross families. JJL performed data analysis of QTLs data and drafted the manuscript. PC conducted interpretation of genome survey data. PL and JJW were involved in traits measure and data analysis. All authors read and approved the final manuscript.

\section{Funding}

This research was supported by the National Key R \& D Program of China (2018YFD0901302), National Nature Science Foundation of China (31472275), Modern Agro-industry Technology Research System (CARS-48), The program of Shandong Leading Talent (LNJY2015002) and Qingdao Industrial Development Program (Science and Technology Benefit Special Project) (17-3-3-62-nsh). The funding body of Dr. Jitao Li designed this study and wrote the manuscript. The funding body of Prof. Jian Li built and collected the Backcross families for genetic linkage map construction.

\section{Availability of data and materials}

The datasets supporting the findings of this article are included within the article and its supplementary information files. The raw sequence data from this study were deposited at the NCBI Nucleotide database with the accession Number QUOF000000000.1.

\section{Ethics approval and consent to participate}

All experimental procedures were approved by the Animal Care and Use Committee at the Institute of Yellow Sea Fisheries Research Institute, Chinese Academy of Fishery Sciences. 


\section{Consent for publication}

Not applicable.

\section{Competing interests}

The authors of Jianjian Lv, Ping Liu, Ping Chen and Jiajia Wang are all the members of the funding projects of Jitao Li and Jian Li. The authors declare that they have no competing interests.

Received: 25 April 2019 Accepted: 16 July 2019

Published online: 22 July 2019

\section{References}

1. Xu WJ, Xie JJ, Shi H, Li CW. Hematodinium infections in cultured ridgetail white prawns, Exopalaemon carinicauda, in eastern China. Aquaculture. 2010;1-4:25-31

2. Li JT, Han JY, Chen P, Chang ZQ, He YY, Liu P, et al. Cloning of a heat shock protein 90 (HSP90) gene and expression analysis in the ridgetail white prawn Exopalaemon carinicauda. Fish Shellfish Immunol. 2012;32:1191-7.

3. Lande R, Thompson R. Efficiency of marker-assisted selection in improvement of quantitative traits. Genetics. 1990;124:743-56.

4. Soller M, Beckmann JS. Restriction fragments length polymorphisms and genetic improvement. Proc. 2nd world Cong. Genet. Appl. Livest. Prod. 1982;6:396-404.

5. Yue GH. Recent advances of genome mapping and marker-assisted selection in aquaculture. Fish Fish. 2013:15:376-96.

6. Andriantahina F, Liu X, Huang H. Genetic map construction and quantitative trait locus (QTL) detection of growth-related traits in Litopenaeus vannamei for selective breeding applications. PLoS One. 2013;8:e75206.

7. Song WT, Li YZ, Zhao YW, Liu Y, Niu YZ, Pang RY, et al. Construction of a high-density microsatellite genetic linkage map and mapping of sexual and growth-related traits in half-smooth tongue sole (Cynoglossus semilaevis). PLoS One. 2012;7:e52097.

8. Li Y, Liu SK, Qin ZK, Geoff W, Wang RJ, Sun LY, et al. Construction of a highdensity, high-resolution genetic map and its integration with BAC-based physical map in channel catfish. DNA Res. 2015;22(1):39-52.

9. Shao CW, Niu YC, Rastas P, Liu Y, Xie ZY, Li HD, et al. Genome-wide SNP identification for the construction of a high-resolution genetic map of Japanese flounder (Paralichthys olivaceus): applications to QTL mapping of Vibrio anguillarum disease resistance and comparative genomic analysis. DNA Res. 2015;22(2):161-70.

10. Peng $W Z$, Xu J, Zhang Y, Feng JX, Dong CJ, Jiang LK, et al. An ultra-high density linkage map and QTL mapping for sex and growth-related traits of common carp (Cyprinus carpio). Sci Rep. 2016;6:26693.

11. Sundin K, Brown KH, Drew RE, Nichols KM, Wheeler PA, Thorgaard GH. Genetic analysis of a development rate QTL in backcrosses of clonal rainbow trout, Oncorhynchus mykiss. Aquaculture. 2005;1-4:75-83.

12. Wang CM, Bai ZY, He XP, Lin G, Xia JH, Sun F, et al. A high-resolution linkage map for comparative genome analysis and QTL fine mapping in Asian seabass, Lates calcarifer. BMC Genomics. 2011;12:174.

13. Reid D, Szanto A, Glebe B, Danzmann R, Ferguson M. QTL for body weight and condition factor in Atlantic salmon (Salmo salar): comparative analysis with rainbow trout (Oncorhynchus mykiss) and Arctic charr (Salvelinus alpinus). Heredity. 2005;94:166-72.

14. Fuji KHO, Honda K, Kumasaka K, Sakamoto T, Okamoto N. Marker-assisted breeding of a lymphocystis disease-resistant Japanese flounder (Pralichthys olivaceus). Aquaculture. 2007;1-4:291-5.

15. Shirak A, Seroussi E, Cnaani A, Howe AE, Domokhovsky R, Zilberman N, et al. Amh and Dmrta2 genes map to tilapia (Oreochromis spp.) linkage group 23 within quantitative trait locus regions for sex determination. Genetics. 2006; 174:1573-81.

16. Palaiokostas C, Bekaert M, Davie A, Cowan ME, Oral M, Taggart JB, et al. Mapping the sex determination locus in the Atlantic halibut (Hippoglossus hippoglossus) using RAD sequencing. BMC Genomics. 2013;14:566.

17. Li YT, Dierens L, Byrne K, Miggiano E, Lehnert S, Nigel P, et al. QTL detection of production traits for the Kuruma prawn Penaeus japonicus (bate) using AFLP markers. Aquaculture. 2006;258:198-210.

18. Wang WJ, Tian Y, Kong J, Li X, Liu X, Yang CH. Integration genetic linkage map construction and several potential QTLs mapping of Chinese shrimp (Fenneropenaeus chinensis) based on three types of molecular markers. Genetika. 2012;48(4):508-21.
19. Baranski M, Gopikrishna G, Robinson NA, Katneni VK, Shekhar MS, Shanmugakarthik J, et al. The development of a high density linkage map for black tiger shrimp (Penaeus monodon) based on CSNPs. PLoS One. 2014; 9:e85413.

20. Yu Y, Zhang XJ, Yuan JB, Li FH, Chen XH, Zhao YZ, et al. Genome survey and high-density genetic map construction provide genomic and genetic resources for the Pacific white shrimp Litopenaeus vannamei. Sci Rep. 2015;5:15612.

21. Liu L, Li J, Liu P, Zhao FZ, Gao BQ, Du Y. A genetic linkage map of swimming crab (Portunus trituberculatus) based on SSR and AFLP markers. Aquaculture. 2012;344:66-81.

22. Cui ZX, Hui M, Liu Y, Song CW, Li XH, Li Y, et al. High-density linkage mapping aided by transcriptomics documents ZW sex determination system in the Chinese mitten crab Eriocheir sinensis. Heredity. 2015;115:206-15.

23. Sun XW, Liu DY, Zhang XF, Li WB. Liu Hui, Hong WG, et al. SLAF-seq: an efficient method of large-scale De Novo SNP discovery and genotyping using high-throughput sequencing PLoS One. 2013;8:e58700.

24. Zhang $Y X$, Wang $L H$, Xin HG, et al. Construction of a high-density genetic map for sesame based on large scale marker development by specific length amplified fragment (SLAF) sequencing. BMC Plant Biol. 2013;13:141.

25. Li B, Tian L, Zhang JY, Huang L, Han FX, Yan SR, et al. Construction of a high-density genetic map based on large-scale markers developed by specific length amplified fragment sequencing (SLAF-seq) and its application to QTL analysis for isoflavone content in Glycine max. BMC Genomics. 2014;15:1086.

26. Qi ZM, Huang L, Zhu RS, Xin DW, Liu CY, Han X, et al. A high-density genetic map for soybean based on specific length amplified fragment sequencing. PLoS One. 2014;9:e114349.

27. Wei QZ, Wang YZ, Qin XD, Zhang YX, Zhang ZT, Wang J, et al. An SNPbased saturated genetic map and QTL analysis of fruit-related traits in cucumber using specific-length amplified fragment (SLAF) sequencing. BMC Genomics. 2014;15:1158.

28. Wang $C M$, Zhu ZY, Lo LC, Feng F, Lin G, Yang WT, et al. A microsatellite linkage map of barramundi, Lates calcarifer. Genetics. 2007;175(2):907-15.

29. Wang L, Chen SL, Tian YS, Deng H. Genetic structure analysis for the backcross, inbreeding and parents family of Japanese flounder Paralichthys olivaceus by microsatellite markers. Prog Fish Sci. 2011;3:38-43 (In Chinese).

30. Yuan JB, Gao Y, Zhang XJ, Wei JK, Liu CZ, Li FH, et al. Genome Sequences of Marine Shrimp Exopalaemon carinicauda Holthuis Provide Insights into Genome Size Evolution of Caridea. Mar Drugs. 2017;15(7):213.

31. Chow S, Dougherty WJ, Sandifer PA. Meiotic chromosome complements and nuclear-DNA contents of 4 species of shrimps of the genus penaeus. J Crustacean Biol. 1990;10:29-36.

32. Kenny NJ, Sin YW, Shen XZ. Genomic sequence and experimental tractability of a new decapod shrimp model, Neocaridina denticulata. Mar Drugs. 2014;12:1419-37.

33. Song LS, Bian C, Luo YJ, Wang LL, You XX, Li J, et al. Draft genome of the Chinese mitten crab, Eriocheir sinensis. GigaScience. 2016;5:5.

34. Lv JJ, Gao BQ, Liu P, Li J, Meng XL. Linkage mapping aided by de novo genome and transcriptome assembly in Portunus trituberculatus: applications in growth-related QTL and gene identification. Sci Rep. 2017;7:7874.

35. Gjerde B, Gjedrem T. Estimates of phenotypic and genetic parameters for carcass traits in Atlantic salmon and rainbow trout. Aquaculture. 1984;36:97-110.

36. Jiao WJ, Fu XT, Dou JZ, Li HD, Su HL, Mao JX, et al. High-resolution linkage and quantitative trait locus mapping aided by genome survey sequencing: building up an integrative genomic framework for a bivalve mollusc. DNA Res. 2014;21:85-101.

37. Liu HY, Fu BD, Pang MX, Feng X, Yu XM, Tong JG. A high-density genetic linkage map and QTL fine mapping for body weight in crucian carp (Carassius auratus) using 2b-RAD sequencing. G3. 2017;7(8):2473-87.

38. Berthier-Schaad Y, Kao WH, Coresh J, Zhang L, Ingersoll RG, Stephens R, et al. Reliability of high-throughput genotyping of whole genome amplified DNA in SNP genotyping studies. Electrophoresis. 2007;28:2812-7.

39. Li JT, Li J, Chen P, Liu P, He YY. Transcriptome analysis of eyestalk and hemocytes in the ridgetail white prawn Exopalaemon carinicauda: assembly, annotation and marker discovery. Mol Biol Rep. 2015;42:135-47.

40. Santiago J, Sturgill TW. Identification of the $\mathbf{S 6}$ kinase activity stimulated in quiescent brine shrimp embryos upon entry to preemergence development as p70 ribosomal protein S6 kinase: isolation of Artemia franciscana p70 $56 \mathrm{k}$ cDNA. Biochem Cell Biol. 2001;79(2):141-52.

41. Sahul Hameed AS, Sarathi M, Sudhakaran R, Balasubramanian G, Syed Musthaq S. Quantitative assessment of apoptotic hemocytes in white spot syndrome 
virus (WSSV)-infected penaeid shrimp, Penaeus monodon and Penaeus indicus, by flow cytometric analysis. Aquaculture. 2006;256(1-4):111-20.

42. Laoong-u-thai Y, Zhao B, Phongdara A, Yang JZ. Molecular characterizations of a novel putative DNA-binding protein LVDBP23 in marine shrimp Letopenaeus vannamei tissues and molting stages. PLoS One. 2011;6(5):e19959.

43. Li H, Handsaker B, Wysoker A, Fennell T, Ruan J, Homer N, et al. The sequence alignment/map format and SAMtools. Bioinformatics. 2009;25:2078-9.

44. Liu DY, Ma CX, Hong WG, Huang L, Liu M, Liu H, et al. Construction and analysis of high-density linkage map using high-throughput sequencing data. PLoS One. 2014;9:e98855.

45. Patel RK, Jain M. NGS QC toolkit: a toolkit for quality control of next generation sequencing data. PLoS One. 2012;2:e30619.

46. Li RQ, Li YR, Kristiansen K, Wang J. SOAP: short oligonucleotide alignment program. Bioinformatics. 2008;24:713-4.

47. Zhang J, Zhang QX, Cheng T, Yang W, Pan HT, Zhang JJ, et al. High-density genetic map construction and identification of a locus controlling weeping trait in an ornamental woody plant (Prunus mume Sieb. Et Zucc). DNA Res. 2015;22(3):183-91.

48. McKenna A, Hanna M, Banks E, Sivachenko A, Cibulskis K, Kernytsky A, et al. The genome analysis toolkit: a MapReduce framework for analyzing nextgeneration DNA sequencing data. Genome Res. 2010;20:1297-303.

49. Wu Y, Bhat PR, Close TJ, Lonardi S. Efficient and accurate construction of genetic linkage maps from the minimum spanning tree of a graph. PLoS Genet. 2008:4:e1000212.

50. Van Os H, Stam P, Visser RG, van Eck HJ. SMOOTH: a statistical method for successful removal of genotyping errors from high-density genetic linkage data. Theor Appl Genet. 2005;112:187-94.

51. Grabherr MG, Haas BJ, Yassour M, Levin JZ, Thompson DA, Amit I, et al. Fulllength transcriptome assembly from RNA-Seq data without a reference genome. Nat Biotechnol. 2011;29:644-52.

52. Pertea G, Huang XQ, Liang F, Antonescu V, Sultana R, Karamycheva S, et al. TIGR gene indices clustering tools (TGICL): a software system for fast clustering of large EST datasets. Bioinformatics. 2003;19:651-2.

53. Stanke M, Waack S. Gene prediction with a hidden Markov model and a new intron submodel. Bioinformatics. 2003:19(2):215-25.

54. Krzywinski M, Schein J, Birol I, Connors J, Gascoyne R, Horsman D, et al. Circos: an information aesthetic for comparative genomics. Genome Res. 2009;19:1639-45.

55. Chen N. Using RepeatMasker to identify repetitive elements in genomic sequences. Curr Protoc Bioinformatics. 2004;4:4.10.

56. Ooijen JWW, Boer MP, Jansen RC, Maliepaard C. MapQTL 4.0: Software for the calculation of QTL positions on genetic maps (user manual). Order-a Journal on the Theory of Ordered Sets \& Its Applications. 2000;501:2412-21.

57. Ooijen JWV. Accuracy of mapping quantitative trait loci in autogamous species. Theor Appl Genet. 1992;84:803-11.

\section{Publisher's Note}

Springer Nature remains neutral with regard to jurisdictional claims in published maps and institutional affiliations.

Ready to submit your research? Choose BMC and benefit from:

- fast, convenient online submission

- thorough peer review by experienced researchers in your field

- rapid publication on acceptance

- support for research data, including large and complex data types

- gold Open Access which fosters wider collaboration and increased citations

- maximum visibility for your research: over $100 \mathrm{M}$ website views per year

At $\mathrm{BMC}$, research is always in progress.

Learn more biomedcentral.com/submissions 